Lau, Ching

[35]

\section{Identification of a new fusion of ETV6/TEL and NTRK3/TRKC in a primitive neuroectodermal tumor}

\author{
Xiao-Nan Li, Charles Harris, Xin-Yan Lu, Pulivarthi Rao \\ $\&$ Ching Lau
}

Texas Children's Cancer Center, Baylor College of Medicine, Houston, Texas, USA

ETV6/TEL, a member of the ETS family of transcription factors, has been involved in a variety of gene fusions in various neoplasms. One of its fusion partners is NTRK3/TRKC, which encodes the receptor for neurotrophin-3. Although NTRK3 is expressed broadly in neural tissues during the growth and development of the central nervous system, its fusion with $T E L$ has never been reported in central nervous system tumors. We report here a new TEL-NTRK3 fusion in a primitive neuroectodermal tumor (PNET). The patient was five months old when she underwent a resection of a left frontal tumor that was diagnosed as a benign desmoplastic infantile ganglioglioma. Three months later she had another resection for the recurrent tumor, which was diagnosed as a highly malignant PNET. G-banding karyotype analysis showed 46, XX in both tumors, but spectral karyotyping revealed a single near-tetraploid cell in the first tumor with a $t(12 ; 15)(\mathrm{p} 13 ; \mathrm{q} 25)$ translocation and the same translocation in all metaphases in the PNET. Subsequent fluorescence in situ hybridization analysis using a $T E L$ probe revealed the translocation of the TEL gene to the der(15) chromosome. Based on a similar translocation previously described in infantile fibrosarcoma, congenital mesoblastic nephroma and acute myeloid leukemia involving the TEL and NTRK3 genes, we used the polymerase chain reaction with reverse transcription, with primers flanking the coding sequences of TEL and NTRK3, to detect a chimeric message of 1.6 kilobases. We located the breakpoint at the end of exon 4 of TEL in frame with exon 12 of NTRK3. This results in a unique TEL-NTRK3 fusion transcript with the helix-loop-helix dimerization domain of TEL fused to the protein tyrosine kinase domain of NTRK3. Since $t(12 ; 15)$ is the only structural chromosome aberration in this case and a similar TEL-NTRK3 fusion product has been used to transform NIH $3 \mathrm{~T} 3$ cells, we propose that the TEL-NTRK3 fusion may have contributed to the cellular transformation and progression of this tumor.

Lee, Ann [36]

\section{Identification of genes differentially expressed in breast cancer cells treated with tamoxifen, using microarray-based expression profiling}

Ann Lee, Anand Krishnasamy, Richard Epstein \& Ga Sze Hong

Division of Medical Sciences, National Cancer Centre, Singapore 169610

Adjuvant tamoxifen therapy is effective in reducing the risk of recurrence and death in patients with estrogen-receptor-positive breast cancer. However, the development of acquired resistance to tamoxifen limits its clinical effectiveness. To determine the effects of tamoxifen on breast cancer cells, long-term cultures of the breast cancer MCF-7 cell line, treated with tamoxifen, were established. Gene expression profiles of tamoxifen-treated and untreated MCF-7 cells were characterized using the Clontech Atlas human cancer 1.2 array, representing 1,176 genes. RNA was extracted from tamoxifen-sensitive cells 4 days and 6 weeks after commencement of treatment with tamoxifen, as well as from untreated MCF-7 cells at the same time points. Only gene expression differences greater than twofold were considered. At 4 days, 13 genes were found to be overexpressed and 2 genes were underex- pressed in the tamoxifen-treated MCF-7 cells compared with the untreated MCF-7 cells. In the tamoxifen-treated MCF-7 cells harvested at 6 weeks, 16 genes were overexpressed and 5 genes were underexpressed compared with the treated cells harvested at 4 days. Wnt-5a, disheveled homologue 1, cyclin kinase inhibitor p19INK4D and the signaling lymphocytic activation molecule were overexpressed in both of the comparisons. Gene expression profiling is useful for the rapid identification of genes differentially expressed during tamoxifen treatment.

Leszczynski, Dariusz

[37]

\section{Proteomics: new way to to determine possible biological effects of mobile phone radiation}

Dariusz Leszczynski \& Sakari Joenväärä

Radiobiology Laboratory, STUK-Radiation and Nuclear Safety Authority, Helsinki, Finland

Despite years of research, the question of whether exposure to radiofrequencymodulated electromagnetic fields (RF-EMF) generated by mobile phones affects human health remains unsolved. We obtained a comprehensive overview of the possible extent of cellular response to RF-EMF irradiation by determining the total cellular changes in protein expression and in protein phosphorylation that occur in response to RF-EMF exposure under athermal conditions. As a model we used human endothelial cell line EA.hy926. Cells were exposed for one hour to a 900-MHz GSM signal. Immediately following exposure we harvested cells and extracted and separated proteins using two-dimensional electrophoresis. To determine changes in protein phosphorylation, ${ }^{32} \mathrm{P}$ was present in the cultures during the exposure period. Using Bio-Rad's PDQUEST 6.1.0 software we identified over 1,200 proteins in two-dimensional gels $(10 \times 20 \mathrm{~cm})$. A large number of protein spots changed expression following irradiation. In control cells we detected over 180 phosphoproteins. RF-EMF exposure has generated a large number of newly phosphorylated proteins that were not present in controls. Among the proteins with altered phosphorylation levels were shock proteins, such as hsp27. Thus the expression and phosphorylation of a large number of proteins isolated from EA.hy926 cells seems to be altered by short RF-EMF exposure, suggesting that cells mount a vigorous response to RF-EMF stress. However, whether the observed stress can cause long-lasting physiological effects remains to be determined.

Lin, Lin

[38]

Identification and characterization of a
$19 q 12$ amplicon in esophageal
adenocarcinomas using tw 0-dimensional
genomic scanning and amplification
mapping of sequence tagged sites

Lin Lin ${ }^{1}$, Michael Prescott ${ }^{1}$, Rork Kuick ${ }^{2}$, Samir Hanash ${ }^{2}$, Mark Orringer ${ }^{1}$, Thomas Glover ${ }^{2,3}$ \& David Beer ${ }^{1}$

${ }^{1}$ Department of Surgery, University of Michigan Medical School, Ann Arbor, Michigan 48109, USA

${ }^{2}$ Department of Human Genetics, University of Michigan Medical School, Ann Arbor, Michigan 48109, USA

${ }^{3}$ Department of Pediatrics, University of Michigan Medical School, Ann Arbor, Michigan 48109, USA

Amplification of genomic DNA can confer a selective advantage in tumors by increasing the dosage of gene(s) involved in tumor development or progression. 


\section{Abstracts: Session II}

We have used a two-dimensional restriction landmark genomic scanning technique to identify gene amplification events in esophageal adenocarcinomas. We cloned a multicopy DNA fragment from a tumor two-dimensional gel and confirmed genomic amplification encompassing this fragment by Southern blot analysis. The corresponding DNA sequence was searched by BLAST, which allowed the use of an electronic polymerase chain reaction to map the amplicon to 19q12. We then characterized the amplicon using sequence-tagged site amplification mapping, an approach recently developed in our laboratory. DNAs from 65 esophageal and 11 gastric cardia adenocarcinomas and their normal controls were investigated using 11 sequence-tagged site markers neighboring the cloned fragment. The amplicon, spanning 8 centimorgans, was narrowed to a minimal region of $0.8 \mathrm{cM}$, which includes the cyclin $\mathrm{E}$ gene. We assayed 14 expressed sequence tags covering the minimal region for gene overexpression. Both DNA amplification and messenger RNA overexpression were observed in 7 of the 14 expressed sequence tags selected. Among them, cyclin E demonstrated the highest frequency of gene amplification and overexpression in the tumors examined. After analyzing the sequencetagged site amplification patterns within BAC contig sequences located at 19q12 in the databases, we further defined the core amplified domain to a region 300 kilobases long. We observed amplification of 19q12 in 13.8\% of esophageal adenocarcinomas. Our study is the first to map the core amplified domain of the 19q12 amplicon physically to a $300-\mathrm{kb}$ region, and the data indicate that cyclin $\mathrm{E}$ is the probable target gene selected by the amplification event at $19 \mathrm{q} 12$.

Linder, Stig

\section{Classification of human ovarian tumors using multivariate data analysis of polypeptide expression patterns}

\author{
Ayodele Alaiya ${ }^{1}$, Bo Franzen ${ }^{2}$, Anders Hagman ${ }^{2}$, Stig Linder ${ }^{1}$ \\ $\&$ Gert Auer $^{1}$ \\ ${ }^{1}$ Department of Oncology-Pathology, Cancercentrum Karolinska, Karolinska \\ Hospital, S-17176 Stockholm, Sweden \\ ${ }^{2}$ Preclinical R\&D, Astra Arcus AB, Södertälje, Sweden
}

Quantitative molecular variation may be used for the development of methods for tumor classification. We used the statistical concept of principal component analysis to type ovarian tumors. We purified tumor cells from ovarian tumors and subjected them to two-dimensional gel electrophoresis. Using a data set derived from the quantitation of 170 polypeptides, we established a model (learning set) with 22 tumors for classification into three groups (benign, borderline and malignant) and then used 18 tumors to test the model. We correctly classified six of eight carcinomas and three of four borderline tumors. Of six benign lesions, two were correctly classified, three were classified as borderline and one was classified as a carcinoma. It may be possible to classify tumors by their constitutive gene expression profile using multivariate analysis.
Ling, Zhang

[40]

\section{Cloning and analyzing $5^{\prime}$ flanking sequences of a tissue-specifically expressed gene derived from mouse nasopharynx}

\author{
Ling Zhang, Kaitai Yao, Ke Lan, Hong Li, Xiangling Feng, \\ Zhiwei He \& Weinong Han
}

Key Laboratory of Carcinogenesis, Chinese Ministry of Public Health, Cancer Research Institute, Central South University, Changsha 410078, China

To construct an animal model of nasopharygeal carcinoma with a nasopharynxspecific regulator, we cloned the gene $\mathrm{YH}$, which is specifically and highly expressed in human nasopharynx and trachea, through scanning high-density gene filtering. A BLAST screen against GenBank showed that this gene is a close homologue to the PLNUC gene, which is strictly expressed in mouse embryonic palate, nasal epithelium and lung and in the trachea of the adult mouse. The same result was obtained by using the polymerase chain reaction with reverse transcription. We cloned and sequenced the $5^{\prime}$ flanking sequence of PLUNC with a GenomeWalker kit; this new sequence has been submitted to GenBank (No. 225964). Its promoter activity was confirmed by a luciferase report gene test. The core promoter was 200 base pairs upstream from the transcription starting site. We constructed a green fluorescent protein expression vector with the new promoter, and it showed a specific expression profile in a transgenic Xenopus model. We are now using this promoter to induce the expression of the $E B V-B N L F 1$ gene, and we will transfer this vector to mice by microinjection of fertilized pronuclear eggs.

Lingen, Mark

[41]

\section{Molecular profiling of anti-angiogenic agents}

Edith I. Cline ${ }^{1}$, Silvio Bicciato ${ }^{2} \&$ Mark Lingen $^{1}$

${ }^{1}$ Department of Pathology, Cardinal Bernadin Cancer Center, Loyola University Medical Center, Maywood Illinois 60153, USA

${ }^{2}$ Institute of Industrial Chemistry, University of Padua, Padua, Italy

Angiogenesis, the growth of new blood vessels from pre-existing ones, is an essential phenotype for tumor formation and progression, and therefore the inhibition of angiogenesis is an attractive means of chemoprevention and chemotherapy. Approximately 20 inhibitors of angiogenesis are under investigation in clinical trials, and other agents with various biological activities have shown great promise. However, the molecular mechanisms by which these agents work are largely unknown. In order to begin to define specific molecular mechanisms of action, we have used complementary DNA microarray technology to determine gene expression profiles of endothelial cells treated with different inhibitors of angiogenesis. We treated human microvascular endothelial cells with either retinoic acid, interferon- $\alpha$, a synthetic analogue of fungal protein (TNP470), thrombospondin-1, or pigmented epithelium-derived factor for $4 \mathrm{~h}$. We isolated RNA, labeled it and used it to probe nylon microarray filters that contained a total of 9,154 genes $(5,295$ named and 3,889 expressed sequence tags). Of these, 205 showed threefold or greater induction or repression. Agglomerative clustering of these genes among the five agents tested resulted in 20 clusters, with each agent behaving differently. Principal component analysis of the expression data also revealed a close similarity of expression patterns between thrombospondin-1 and TNP470. Data detailing changes in expression patterns of all the agents will also be presented. This work demonstrates not only the possibility of obtaining potential molecular profiles of anti-angiogenic agents, but also an opportunity for increased efficacy and reduced toxicity during the development of drugs based on these agents. 